\title{
Widget-based Alarm Recognition and a Management Support Interface System in Nuclear Power Plants
}

\author{
Seung Min Lee*, Hana Park, Gwi Sook Jang, Inkoo Hwang \\ Korea Atomic Energy Research Institute, South Korea \\ * jewellee@kaeri.re.kr
}

\begin{abstract}
As small modular reactors (SMRs) and various computerized operating support systems have been developed, visual display unit(VDU)-based multifunctional consoles have been proposed. The more a console depends on VDUs, the more human system interface (HSI) designers need to consider the decrease in the human performance caused by the effect of navigation tasks. This paper proposes a widget-based alarm recognition and management support interface system to increase the operator's ability to recognize alarms and to manage alarm-related tasks by reducing additional and excessive navigation tasks. With a small display screen, the system provides operators with quick and accurate access to alarm-related information and allows them to make corrective responses.
\end{abstract}

Keywords-Human-System Interface, Human Performance, Information display, Monitoring system

\section{INTRODUCTION}

$\mathrm{T}$ HE change from analogue-based information display panels in the main control rooms (MCRs) in Nuclear Power Plants (NPPs) to digital-based visual display units (VDUs) has brought accompanying changes in the operating environment and operators' tasks.

VDUs and various computerized operating support systems provide an enormous amount of information and facilitate operators' performance of their monitoring and control tasks. However, displaying a large amount of information on the limited VDU screen generates a major secondary task: navigating. Navigation produces a decrease in performance as the operator struggles to access the correct information pages. In a study on the cause of human errors that occur in a digitalized MCR, the task of navigating was pointed out as the second highest human error factor [1-3]. Navigation increases work time, interrupts accurate and rapid operation, and decreases human performance. Thus, the designing of the graphic user interface (GUI) in display screen is an important and necessary consideration in studying the effect of the navigating task.

With the development of small modular reactors (SMRs) and various computerized operating support systems, the concept

S. M. Lee is with the Korea Atomic Energy Research Institute, 34057 Daejeon, Korea (e-mail: jewellee@kaeri.re.kr)

H. N. Park is with the Korea Atomic Energy Research Institute, 34057 Daejeon, Korea (e-mail: hnpark@kaeri.re.kr)

G. S. Jang is with the Korea Atomic Energy Research Institute, 34057 Daejeon, Korea (e-mail: gsjang@kaeri.re.kr)

I. K. Hwang is with the Korea Atomic Energy Research Institute, 34057 Daejeon, Korea (e-mail: ikhwang@kaeir.re.kr)

(C) The Authors, published by EDP Sciences. This is an open access article distributed under the terms of the Creative Commons Attribution License 4.0 (http://creativecommons.org/licenses/by/4.0/) of local control stations and multifunctional consoles emerged as shown in Figs. 1 and 2.

In such a console, an operator is responsible for one reactor unit or one small plant; thus, he/she needs to access all the monitoring information and to conduct appropriate control activity.

The console is quite dependent on the VDU, and human system interface (HSI) designers must consider the navigation tasks. In other words, they have to minimize the navigation tasks or support performance of navigation tasks. Reducing navigation tasks, especially finding alarm-related information, is necessary because the alarm-related tasks need to be dealt with in real-time.

This paper proposes an alarm recognition and management support interface system. The proposed interface effectively provides the alarm information by efficiently utilizing a small screen space. The system will aid rapid recognition and accurate response, and thus improve the human operators' performance.

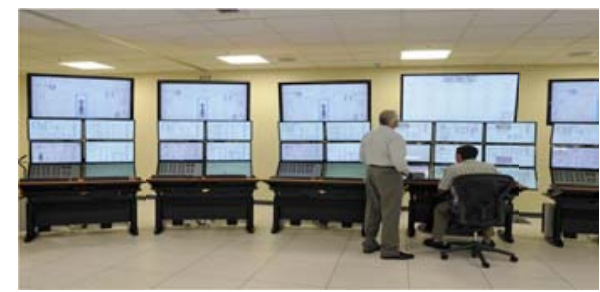

Fig. 1. Local control stations of the SMR
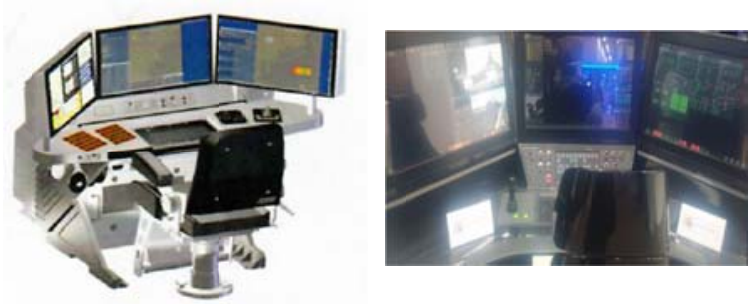

Fig. 2. Multifunctional control stations

\section{ALARM DISPLAY SYSTEMS IN NPPS}

To design the GUIs of the proposed interface system, we analyzed the characteristics of the existing alarm display screens in conventional and advanced MCRs and considered the pros and cons of existing alarm display screens.

- Characteristics of analogue-based alarm panels (Fig. 3)

- Alarm tiles for similar subsystems are gathered and located and fixed in the upper space of the panel. It helps operators to observe the real status of plants 
quickly, accurately and continuously.

- Alarm tiles generate visual and auditory stimuli in real time when alarms occur. The stimuli also make it possible for the human operators to response quickly and to increase the situation awareness of the status of plants.

- The space for displaying alarms is limited, and operators do not have enough immediate information on information that is not displayed at the moment.

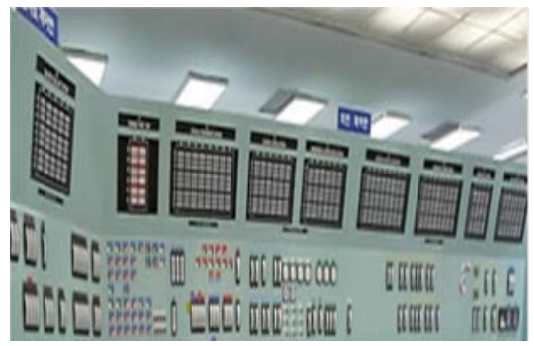

Fig. 3. Analogue-based alarm display panels in the conventional MCRs

- $\quad$ Characteristics of digitalized alarm screens (Figs.4 and 5)

- All alarm data and modified information, such as collecting alarm information on similar subsystems, collecting it on the same priority, and listing it in sequence of occurrence, is displayed on the screen through the computerized function.

- The operators can obtain various kinds of information that the display system provides; however, they are required to conduct navigation tasks to access the right information among the enormous hierarchical information structure, which decreases the efficiency of accessing information rapidly and accurately and understanding the status of plants.

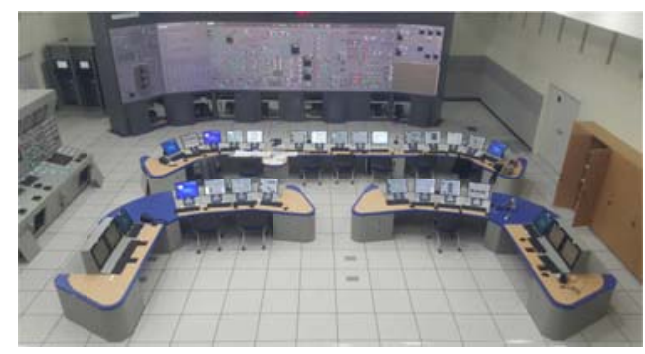

Fig. 4. The advanced MCR

\section{WIDGET-BASED ALARM RECOGNITION MANAGEMENT INTERFACE SYSTEM (WARMI)}

Based on our analysis of the existing alarm systems, we isolated the advantages of the existing alarm system. The GUI of the WARMI was designed by reflecting and improving the selected advantages.

- Selected characteristics of the existing alarm system

- Gathering alarms related to each subsystem and prioritizing alarms effectively improves rapid and accurate recognition.

- Showing newly generated alarms and deleting acknowledged or processed alarms makes it possible to utilize the small space efficiently and call operators' attention to alarm recognition.

- Reducing the navigation task load by moving to the right pages is effective eliciting the appropriate

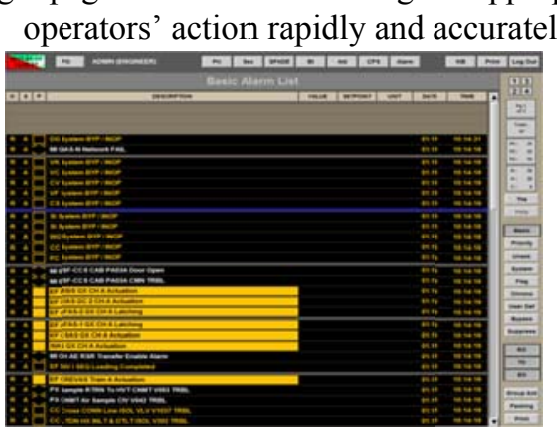

(a) The list type alarm display

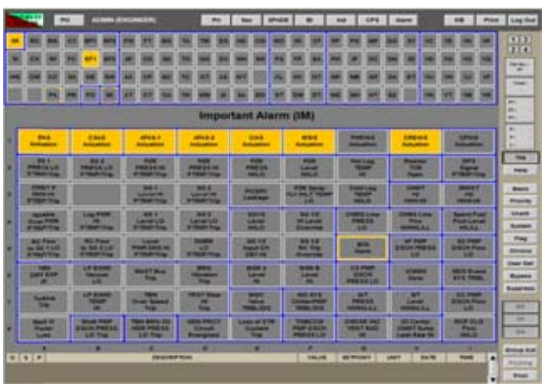

(b) The tile type alarm display

Fig. 5. Digitalized alarm display screens

The structure of the WARMI system and how to use it is described as follows.

- $\quad$ The structure of the WARMI system (Fig. 6)

- The proposed interface was designed using a 10-inch monitor, and operates independently with the existing Information Processing System (IPS) [4] display screen and processors.

- The information displayed in the WARMI screen is based on the alarm information of the IPS, and the information on alarm generation, acknowledgement, blinking and off is moved from the IPS processor to WARMI in only one direction.

- The operator can switch the IPS screen to the desired (alarm-related) subsystem by clicking WARMI alarm icons. The screen switching command only moves in one direction from the WARMI to the IPS.

- The alarm-related operator actions are only available on the IPS screen through the prompt alarm recognition through WARMI and movement to the IPS screen. Human operators' action for alarm through WARMI is not available. The interface provides detailed information about the alarm through each aliarm related icon.

- $\quad$ The operation of the WARMI system (Fig. 7)

- When an alarm occurs, the subsystem related to the generated alarm is expressed by the folder form, and an icon describing the alarm is generated in the folder in WARMI. The intuitive recognition for the information such as the number of generated alarms for each subsystem, and the priority of the alarm is 
availaable.

- By cllicking the folder, the operators get detailed information about the alarms. By clicking the alarm icon, the operators obtain detailed information about the alarm such as the priority, alarm causes, and corrective action to respond the alarm.

- The operators can change the IPS screen page to the related alarm page. It helps operators to directly move to the relevant pages and to perform the relevant operators' actions through the detailed information on the WARMI screen.

- The interface automatically deletes the icon corresponding to an extinguished alarm when the alarm handled by the operator action on the IPS disappears or becomes normal. If all alarm icons are deleted in the folder, the folder is also automatically deleted in WARMI.
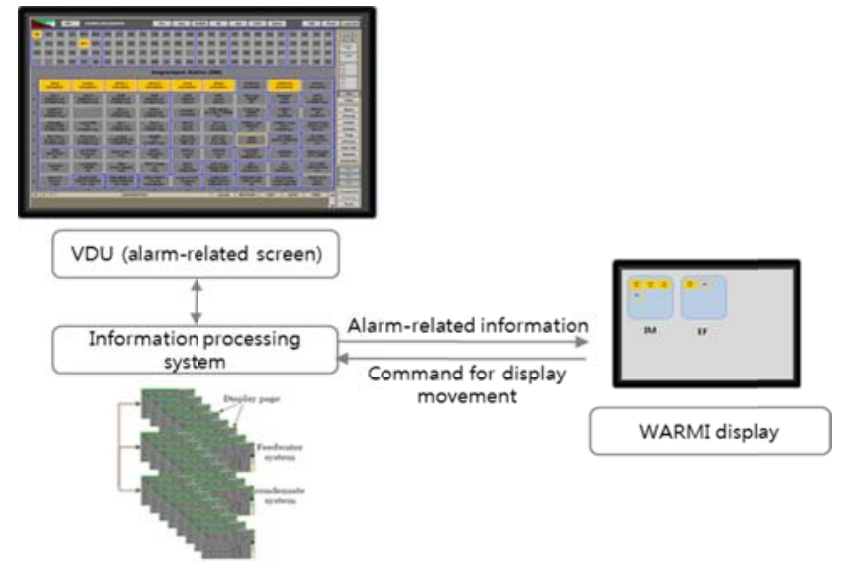

Fig. 6. Structure of WARMI

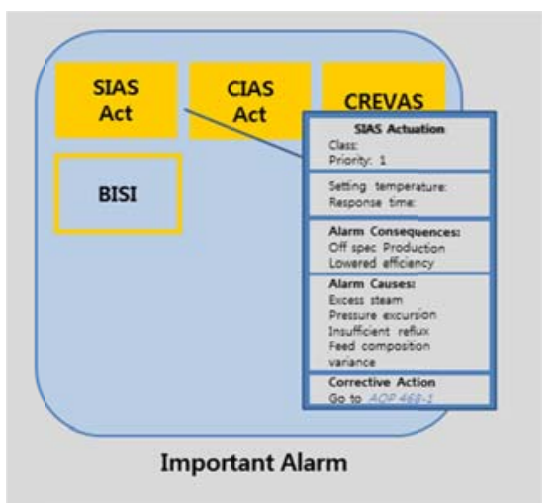

Fig. 7. WARMI graphic user interface

\section{SUMMARY AND FUTURE WORKS}

As small modular reactors (SMRs) and various computerized operating support systems were developed, local control stations and multifunctional consoles, which are very dependent on the VDU were introduced.

To reduce the navigation tasks created by using VDUs, the alarm recognition and management support interface system, WARMI, was proposed to aid rapid recognition and accurate response.

We analyzed the characteristics of the existing alarm display screens on conventional and advanced MCRs and designed the GUI of WARMI.
A test will be conducted to verify the effectiveness of the proposed interface system in terms of human performance.

Using WARMI will lighten the navigation task loads of operators using VDUs and improve their performance on alarm recognition and conducting the appropriate responses.

\section{REFERENCES}

[1] I. S. Jang, W. D. Jung, P. H. Seong, "Human Error and the Associated Recovery Probabilities for Soft Control being Used in the Advanced MCRs on NPPs," Annals of Nuclear Energy, Vol. 87, pp. 290-298, 2016.

[2] O'Hara, J., \& Brown, W. (2002), “"The effects of interface management tasks on crew performance and safety in complex, computer-based systems", NUREG/CR-6633. Washington, D.C.:U.S. NRC.

[3] R. E. Patterson et al., "A Human Cognition Framework for Information Visualization," Computers \& Graphics, Vol. 42, pp. 42-58, 2014.

[4] NPX80-IC-SD710, "System Description for the Data Processing System for Nuplex $80+. "$ 